\title{
The integral form of APS boundary conditions in the Bag Model
}

\author{
A. A. Abrikosov, jr. ${ }^{a *}$, A. Wipf ${ }^{b \dagger}$ \\ a Institute of Theoretical and Experimental Physics \\ B. Cheremushkinskaya 25, Moscow 117 218, Russia \\ ${ }^{b}$ Institute of Theoretical Physics, Friedrich-Schiller University \\ Max-Wien-Platz 1, 07743 Jena, Germany
}

\begin{abstract}
We propose an integral form of Atiah-Patodi-Singer spectral boundary conditions (SBC) and find explicitly the integral projector onto SBC for the 3-dimensional spherical cavity. After discussion of a simple example we argue that the relation between the projector and fermion propagator is universal and stays valid independently of the bag form and space dimension.
\end{abstract}

\section{Introduction}

The two principal problems of QCD are confinement and spontaneous breaking of chiral invariance. Both of them take place in the strongly interacting domain where the theory becomes nonperturbative. Most probably the two are interrelated. However, usually they were considered separately. Up to now the spontaneous chiral invariance breaking (SCIB) was discussed mostly in infinite space. It would be interesting to study specific features of SCIB that appear due to localization of quarks in finite volume. In order to do that we confine the quarks in a chiral invariant way.

One way to lock fermions in a finite volume without spoiling chiral symmetry is to impose the so-called spectral boundary conditions (SBC). They were introduced by Atiyah, Patodi and Singer (APS) who investigated the spectral asymmetry for manifolds with boundaries [5, 6]. Later these boundary conditions were widely applied in studies of anomalies on manifolds with boundaries [7].

Originally the APS boundary conditions were formulated for compact, euclidean spinmanifolds in arbitrary even dimension $2 n$. Such a manifold has a compact $2 n-1$, i. e. odddimensional boundary. However in physical applications one also meets odd-dimensional spatial bags evolving in Euclidean or Minkowsky time. Evolution converts the spatial boundary of such a bag into an infinite space-time cylinder. Recently it was shown 8 that in these problems instead of the full time-dependent boundary conditions on the cylinder one may use static SBC on the spatial boundary of the bag. Thus it turns out

\footnotetext{
*e-mail: persik@itep.ru

${ }^{\dagger}$ e-mail: wipf@tpi.uni-jena.de
} 
that the APS boundary conditions make sense both on odd (the classical case) and even dimensional surfaces (the modified SBC).

The spectral conditions are essentially nonlocal, $i$. e. they are defined on the boundary as a whole. According to the original "constructive" definition all spinor fields are expanded in terms of eigenfunctions of the Dirac operator restricted to the boundary and certain eigenfunctions in this expansion are required to be absent. This ensures Hermiticity of the Dirac operator and charge conservation in the bag.

However the SBC may also be written in an integral form. Summation over separate boundary harmonics gives an integral projection operator (we call it the SBC-projector). The APS conditions state that it must annihilate fermionic wave functions on the boundary. The SBC-projector and its properties are considered in the current paper. We study the modified SBC for the three-dimensional spherical bag. Its boundary is the Riemann sphere $S^{2}$ and the corresponding harmonics may be expressed via spherical spinors. In this case the summation may be carried out and the SBC projection operator may be found explicitly.

A remarkable feature of our result is that the SBC projection operator is immediately related to the fermionic Green function. We claim that this relation is general and holds for arbitrary non-spherical boundaries in any dimension.

The paper has the following structure. We shall review the spectral boundary conditions in Section 1. In Section 2 we shall discuss the APS conditions on the 3-dimensional sphere and calculate the SBC-projector. Then, in Section 3 we shall discuss the relation between the SBC-projector and fermion propagator first for a simple model and then in the general case. These will be followed by a summary and conclusions.

\section{The spectral boundary conditions}

\subsection{Conventions}

First we will introduce coordinates, Dirac matrices and fix a gauge that allows us to formulate spectral boundary conditions. The APS boundary conditions in any even dimension are defined similarly and we may limit ourselves to the 4-dimensional case. Generalization to higher dimensions is straightforward.

Let us consider massless fermions interacting with a gauge field $\hat{A}$ in some Euclidean domain $B$ that may be either a closed 4-dimensional cavity $B_{4}$ or an infinite space-time cylinder $B_{3} \otimes R$. We choose the curvilinear coordinates so that near the boundary $\partial B$ the first coordinate $\xi$ points along the outward normal while the three others, $q^{i}$, parametrize $\partial B$ itself. The origin $\xi=0$ lies on $\partial B$. Following the classics we shall assume that near the surface the metric $g_{\alpha \beta}$ depends only on $q$ so that

$$
d s^{2}=d \xi^{2}+g_{i k}(q) d q^{i} d q^{k}
$$

For some subtleties concerning this point we refer to [9]. We choose the gauge such that on the boundary the normal component $\hat{A}_{\xi}=0$.

At this point it is convenient to choose the Dirac matrix $\gamma^{\xi}$. Let $I$ be the $2 \times 2$ unity matrix. Then

$$
\gamma^{\xi}=\left(\begin{array}{cc}
0 & i I \\
-i I & 0
\end{array}\right) ; \quad \gamma^{q}=\left(\begin{array}{cc}
0 & \sigma^{q} \\
\sigma^{q} & 0
\end{array}\right)
$$


where $\left\{\sigma^{1}, \sigma^{2}, \sigma^{2}\right\}$ are the ordinary Pauli matrices. With these definitions the Dirac operator for massless fermions on the surface takes the form,

$$
-i \not||_{\partial B_{4}}=-i \gamma^{\alpha} \nabla_{\alpha}=\left(\begin{array}{cc}
0 & \hat{M} \\
\hat{M}^{\dagger} & 0
\end{array}\right)=\left(\begin{array}{cc}
0 & I \partial_{\xi}-i \hat{\nabla} \\
-I \partial_{\xi}-i \hat{\nabla} & 0
\end{array}\right)
$$

where $\hat{\nabla}=\sigma^{q} \nabla_{q}$ is the convolution of covariant gradient along the boundary $\nabla_{q}$ with $\sigma$-matrices. Note that Hermitian conjugated operators $\hat{M}$ and $\hat{M}^{\dagger}$ differ only by the sign of $\partial_{\xi}$.

Further on we shall call the linear differential operator $-i \hat{\nabla}$ on the boundary the boundary operator.

$$
\hat{B}_{4}=-i \hat{\nabla}=-i \sigma^{q} \nabla_{q}
$$

It is Hermitian and contains the tangential gauge field $\hat{A}_{q}$ and the spin connection that arises from the curvature of $\partial B_{4}$.

It is well known that since the massless Dirac operator anticommutes with $\gamma^{5}$-matrix,

$$
\left\{-i \nabla, \gamma^{5}\right\}=0, \quad \gamma^{5}=\left(\begin{array}{rr}
I & 0 \\
0 & -I
\end{array}\right)
$$

it preserves helicity of massless quarks. This property is called chiral invariance. In order to retain it in finite space one needs chirally invariant boundary conditions.

\subsection{The APS boundary conditions}

\subsubsection{The classical 4-dimensional SBC}

Atiah, Patodi and Singer investigated spectra of Dirac operator $-i \not \nabla$ on manifolds with boundaries. If we separate upper and lower (left and right) components of 4-spinors the corresponding spectral equation will take the form

$$
-i \not \nabla \psi_{\Lambda}=-i \not\left(\begin{array}{c}
u_{\Lambda} \\
v_{\Lambda}
\end{array}\right)=\Lambda\left(\begin{array}{c}
u_{\Lambda} \\
v_{\Lambda}
\end{array}\right)=\Lambda \psi_{\Lambda} .
$$

The next step in the construction of SBC is to Fourier-expand $u$ and $v$ near the boundary. Let 2 -spinors $e_{\lambda}(q)$ be eigenfunctions of the boundary operator $-i \hat{\nabla}$ :

$$
\hat{B}_{4} e_{\lambda}(q)=-i \hat{\nabla} e_{\lambda}(q)=\lambda e_{\lambda}(q)
$$

Note that the form of this equation and the eigenfunctions $e_{\lambda}(q)$ depend on the gaugefixing. It is here that the gauge condition $\hat{A}_{\xi}(0, q)=0$ becomes important.

The operator $-i \hat{\nabla}$ is Hermitian so that its eigenvalues $\lambda$ are real. The functions $e_{\lambda}$ form an orthogonal basis on $\partial B_{4}$. In principle $-i \hat{\nabla}$ may have zero-modes but for the sphere and convex manifolds this is not the case.

Due to assumption (11) in the vicinity of the boundary the normal coordinate $\xi$ separates and the 2 -spinors $u_{\Lambda}$ and $v_{\Lambda}$ may be expanded in series:

$$
\begin{array}{ll}
u_{\Lambda}(\xi, q)=\sum_{\lambda} f_{\Lambda}^{\lambda}(\xi) e_{\lambda}(q), & f_{\Lambda}^{\lambda}(\xi)=\int_{\partial B_{4}} e_{\lambda}^{\dagger}(q) u_{\Lambda}(\xi, q) \sqrt{g} d^{3} q \\
v_{\Lambda}(\xi, q)=\sum_{\lambda} g_{\Lambda}^{\lambda}(\xi) e_{\lambda}(q), & g_{\Lambda}^{\lambda}(\xi)=\int_{\partial B_{4}} e_{\lambda}^{\dagger}(q) v_{\Lambda}(\xi, q) \sqrt{g} d^{3} q
\end{array}
$$


where $g=\operatorname{det}\left\|g_{i k}\right\|$ is the determinant of the metric on the boundary.

The APS boundary conditions may be defined in two equivalent ways: either in terms of separate harmonics or via an integral operator.

- The traditional form of spectral boundary conditions states that on the boundary, i. e. at $\xi=0$

$$
\begin{array}{llll}
\left.f_{\Lambda}^{\lambda}\right|_{\partial B_{4}}=0 & \text { for } & & \lambda>0 \\
\left.g_{\Lambda}^{\lambda}\right|_{\partial B_{4}}=0 & \text { for } & & \lambda<0
\end{array}
$$

- Another way is to introduce integral projectors $\mathcal{P}_{4}^{+}$and $\mathcal{P}_{4}^{-}$onto boundary modes with positive and negative $\lambda$ (4 means the 4 -dimensional case):

$$
\mathcal{P}_{4}^{+}\left(q, q^{\prime}\right)=\sum_{\lambda>0} e_{\lambda}(q) e_{\lambda}^{\dagger}\left(q^{\prime}\right) ; \quad \mathcal{P}_{4}^{-}\left(q, q^{\prime}\right)=\sum_{\lambda<0} e_{\lambda}(q) e_{\lambda}^{\dagger}\left(q^{\prime}\right)
$$

If we join two-dimensional projectors $\mathcal{P}^{+}$and $\mathcal{P}^{-}$into the $4 \times 4$ integral operator $\mathcal{P}_{4}$ then the SBC for a 4-spinor $\psi$ will look as follows:

$$
\mathcal{P}_{4} \psi(q)=\int_{\partial B_{4}}\left(\begin{array}{cc}
\mathcal{P}_{4}^{+}\left(q, q^{\prime}\right) & 0 \\
0 & \mathcal{P}_{4}^{-}\left(q, q^{\prime}\right)
\end{array}\right)\left(\begin{array}{c}
u\left(q^{\prime}\right) \\
v\left(q^{\prime}\right)
\end{array}\right) \sqrt{g} d^{3} q^{\prime}=0 .
$$

The SBC-projector $\mathcal{P}_{4}$ commutes with $\gamma^{5}$, and this boundary condition by construction respects chiral invariance,

$$
\left[\mathcal{P}_{4}, \gamma^{5}\right]=0
$$

Let us denote by $\mathcal{I}$ the unity operator on the function space spanned by the $e_{\lambda}$. Obviously, because of completeness,

$$
\mathcal{P}_{4}^{+}\left(q, q^{\prime}\right)+\mathcal{P}_{4}^{-}\left(q, q^{\prime}\right)=\mathcal{I}\left(q, q^{\prime}\right)=\left.I \delta\left(q-q^{\prime}\right)\right|_{\partial B_{4}}
$$

where the last expression is the $\delta$-function on the bag surface $\partial B_{4}$.

\subsubsection{The truncated $3+1$-dimensional SBC}

Now let us turn to fermions confined in a 3-dimensional spatial bag $B_{3}$ that evolves in Euclidean time and sweeps the infinite space-time cylinder $B_{3} \otimes R$. We will call the first three coordinates "space" and the fourth one "time". The boundary operator consists of spatial and temporal parts:

$$
\hat{B}_{4}=-i \hat{\nabla}_{\partial B_{3} \otimes R}=-i \hat{\nabla}_{\partial B_{3}}-i \sigma^{z} \partial_{4}=\hat{B}_{3}-i \sigma^{z} \partial_{4}
$$

We will call the spatial part $\hat{B}_{3}=-i \hat{\nabla}_{\partial B_{3}}$ the truncated boundary operator. Let its eigenfunctions be $e_{\lambda}^{ \pm}$(there was no $( \pm)$-superscript in 4-dimensions):

$$
-i \hat{\nabla}_{\partial B_{3}} e_{\lambda}^{ \pm}(q)=\hat{B}_{3} e_{\lambda}^{ \pm}(q)= \pm \lambda e_{\lambda}^{ \pm}(q), \quad \lambda>0
$$

Wave functions on the space-time boundary $\partial B_{3} \otimes R$ can be expanded in $e_{\lambda}^{ \pm}$and longitudinal (temporal) plane waves:

$$
\begin{aligned}
& u_{\Lambda}=\sum_{\lambda>0} \int \frac{d k}{2 \pi} e^{i k t}\left[f_{\Lambda}^{+\lambda, k} e_{\lambda}^{+}+f_{\Lambda}^{-\lambda, k} e_{\lambda}^{-}\right] \\
& v_{\Lambda}=\sum_{\lambda>0} \int \frac{d k}{2 \pi} e^{i k t}\left[g_{\Lambda}^{+\lambda, k} e_{\lambda}^{+}+g_{\Lambda}^{-\lambda, k} e_{\lambda}^{-}\right] .
\end{aligned}
$$


The truncated operator $-i \hat{\nabla}_{\partial B_{3}}$ anticommutes with $\sigma^{z}$ and because of that the temporal term in (14) mixes positive and negative spatial harmonics. This makes the full SBC: a) "future-dependent" and b) hard to handle.

However it was shown in [8] that in this case one may use the simpler truncated APS constraints. In terms of harmonics of the truncated boundary operator they look as follows:

$$
\begin{aligned}
& \left.f_{\Lambda}^{+\lambda, k}\right|_{\partial B_{3}}=0 \\
& \left.g_{\Lambda}^{-\lambda, k}\right|_{\partial B_{3}}=0
\end{aligned}
$$

These conditions are purely spatial and do not depend on time. Thus they cause no problems with causality and may be applied both in Euclidean and Minkowski spaces.

Projectors onto positive and negative boundary harmonics in the $(3+1)$-case are defined in complete analogy with (10):

$$
\mathcal{P}_{3+1}^{ \pm}\left(q, q^{\prime}\right)=\sum_{\lambda>0} e_{\lambda}^{ \pm}(q)\left[e_{\lambda}^{ \pm}\left(q^{\prime}\right)\right]^{\dagger} .
$$

This allows to put the truncated SBC in the integral form as follows:

$$
\mathcal{P}_{3+1} \psi(q)=\int_{\partial B_{4}}\left(\begin{array}{cc}
\mathcal{P}_{3+1}^{+}\left(q, q^{\prime}\right) & 0 \\
0 & \mathcal{P}_{3+1}^{-}\left(q, q^{\prime}\right)
\end{array}\right)\left(\begin{array}{c}
u\left(q^{\prime}\right) \\
v\left(q^{\prime}\right)
\end{array}\right) \sqrt{g} d^{2} q^{\prime}=0,
$$

where the integration runs over the 2-dimensional spatial boundary.

\subsection{The physics of SBC}

It may be proved that SBC are chirally invariant, ensure Hermicity of the Dirac operator and fermion conservation in the bag. The interesting physical property of SBC is that fermionic wave functions may be continued out of the bag in a square integrable way.

Look how this may be shown. Let us write the eigenvalue equations for Dirac operator near the boundary (remember that $\xi$ is the outward spatial normal). In $3+1$-dimensional case harmonics corresponding to $\pm \lambda$ get mixed, therefore for each value of $\lambda$ we get a set of four linked equations (instead of two independent pairs for $k=0$ in $4 d$ ):

$$
\begin{aligned}
\left(\partial_{\xi}+\lambda\right) g_{\Lambda}^{+\lambda, k} & =\Lambda f_{\Lambda}^{+\lambda, k}+i k g_{\Lambda}^{-\lambda, k} ; \\
-\left(\partial_{\xi}-\lambda\right) f_{\Lambda}^{+\lambda, k} & =\Lambda g_{\Lambda}^{+\lambda, k}+i k f_{\Lambda}^{-\lambda, k}: \\
\left(\partial_{\xi}-\lambda\right) g_{\Lambda}^{-\lambda, k} & =\Lambda f_{\Lambda}^{-\lambda, k}-i k g_{\Lambda}^{+\lambda, k} ; \\
-\left(\partial_{\xi}+\lambda\right) f_{\Lambda}^{-\lambda, k} & =\Lambda g_{\Lambda}^{-\lambda, k}-i k f_{\Lambda}^{+\lambda, k} .
\end{aligned}
$$

According to conditions (17) the RHS of equations (20a, 20d) vanish on the boundary. The behaviour of $g^{+}$and $f^{-}$on the boundary is governed by homogeneous equations so that:

$$
\left.\frac{\partial_{\xi} f_{\Lambda}^{-\lambda, k}}{f_{\Lambda}^{-\lambda, k}}\right|_{\xi=0}=\left.\frac{\partial_{\xi} g_{\Lambda}^{+\lambda, k}}{g_{\Lambda}^{+\lambda, k}}\right|_{\xi=0}=-\lambda<0 .
$$

Thus the nonvanishing spinor components $g^{+}$and $f^{-}$have negative logarithmic derivatives at the boundary. Therefore the eigenfunctions may be continued exponentially out of both the $4 d$-bag or the $(3+1) d$-world cylinder in a square integrable way. After the continuation the particles stay located mainly inside the bag. This proves that SBC is a quite natural physical requirement. 


\section{The SBC-projector for sphere}

Now we are going to calculate explicitly the integral SBC-projector for the boundary shaped as an ordinary 3-dimensional Riemann sphere $S^{2}$. Properties of two-dimensional fermions on the sphere were investigated in detail in [10]. The eigenfunctions of Dirac operator in spherical coordinates were found in [11]. However, it is important that the form of the boundary operator and, consequently, of SBC-projector varies depending on the representation of spinors. A proper representation may seriously reduce the complexity of the problem. We deem our real success the implementation of what we call the "work representation". It notably simplifies the task and makes the calculation feasible. This representation will be introduced in the following section. Then we will outline the actual computation and, finally, present the result in covariant form.

\subsection{Transformation of Dirac operator}

The form of the Dirac operator, boundary operator and SBC-projector depend on choice of the $\gamma$-matrices. There exists a coordinate-dependent transformation of spinors that converts the eigenfunctions of the boundary operator on the sphere into conventional spherical spinors. We call this the work representation.

Let us start from standard Cartesian coordinates $x_{\mu}, \mu=1, \ldots 4$. The corresponding $\gamma$-matrices are:

$$
\gamma_{a}=\left(\begin{array}{rr}
0 & \sigma_{a} \\
\sigma_{a} & 0
\end{array}\right), \quad \gamma_{4}=\left(\begin{array}{rr}
0 & i I \\
-i I & 0
\end{array}\right) \quad \text { or } \quad \gamma_{\mu}=\left(\begin{array}{rr}
0 & \sigma_{\mu} \\
\sigma_{\mu}^{\dagger} & 0
\end{array}\right)
$$

with $\sigma_{\mu}=\left(\sigma_{a}, i I\right), \sigma_{\mu}^{\dagger}=\left(\sigma_{a},-i I\right)$. The conventional rotation generators for 4-spinors are (for spatial rotations it is convenient to use generators with one index).

$$
\Sigma_{\mu \nu}=-\frac{i}{2}\left[\gamma_{\mu}, \gamma_{\nu}\right] \quad \text { and } \quad \Sigma^{a}=\frac{1}{2} \epsilon^{a b c} \Sigma_{b c}
$$

The Cartesian Dirac operator is:

$$
-i \not \nabla_{C a r t}=-i \gamma_{\mu} \partial_{\mu}
$$

Transformation to the work representation consists of two independent $\frac{\pi}{2}$-turns. The spatial turn about the radius simplifies the boundary operator but leaves untouched the $\gamma_{r}=\gamma_{a} n_{a}$ matrix $\left(\vec{n}=\frac{\vec{r}}{r}\right)$. The rotation in $\left(r x_{4}\right)$-plane interchanges the $\gamma_{4}$ and $\gamma_{r}$ matrices so that $\gamma_{4}$ points along the radius as required by APS boundary conditions. The product of the two rotations is:

$$
V_{W}=\exp \frac{i \pi}{4} \vec{\Sigma} \vec{n} \exp \frac{i \pi}{4} \Sigma_{a 4} n_{a}=\left(\begin{array}{cc}
\frac{1+i \vec{\sigma} \vec{n}}{\sqrt{2}} & 0 \\
0 & \frac{1+i \vec{\sigma} \vec{n}}{\sqrt{2}}
\end{array}\right)\left(\begin{array}{cc}
\frac{1-i \vec{\sigma} \vec{n}}{\sqrt{2}} & 0 \\
0 & \frac{1+i \vec{\sigma} \vec{n}}{\sqrt{2}}
\end{array}\right)=\left(\begin{array}{cc}
I & 0 \\
0 & i \vec{\sigma} \vec{n}
\end{array}\right)
$$

The result of the transformation on the matrices $\gamma_{4}$ and $\gamma_{r}$ is:

$$
V_{W}^{\dagger} \vec{\gamma} \vec{n} V_{W}=\gamma_{4} ; \quad V_{W}^{\dagger} \gamma_{4} V_{W}=-\vec{\gamma} \vec{n}
$$

After rotation to the work frame the Dirac operator takes the form:

$$
-i \nabla_{W}=-i V_{W}^{\dagger} \nabla_{C a r t} V_{W}=\left(\begin{array}{cc}
0 & \left(\partial_{r}+\frac{1}{r}\right)+\frac{\hat{B}_{W}}{r} \\
-\left(\partial_{r}+\frac{1}{r}\right)+\frac{\hat{B}_{W}}{r} & 0
\end{array}\right)+i \vec{\gamma} \vec{n} \partial_{4},
$$


Up to the term $\frac{1}{r}$ that accompanies the $\partial_{r}$-derivative (it may be eliminated by redefining the wave function $\psi \rightarrow \psi / r)$ this operator has exactly the required form $\left(\gamma_{4}\right.$ is aligned with the radius). Therefore we may project solutions of the Dirac equation onto eigenfunctions of $\hat{B}_{W}$ and impose on them the modified APS-boundary conditions.

\subsection{The boundary operator}

For convenience let us introduce $(3+1)$ cylindrical coordinates:

$$
x^{1}=r \sin \theta \cos \phi ; \quad x^{2}=r \sin \theta \sin \phi ; \quad x^{3}=r \cos \theta ; \quad x^{4}=t .
$$

After the spinor rotation (26) the boundary operator in (27) takes the following form:

$$
\hat{B}_{W}=\left(\begin{array}{cc}
-i \partial_{\phi}+1 & -e^{-i \phi}\left(\partial_{\theta}-i \cot \theta \partial_{\phi}\right) \\
e^{i \phi}\left(\partial_{\theta}+i \cot \theta \partial_{\phi}\right) & i \partial_{\phi}+1
\end{array}\right)=I+2 \hat{L}_{W}^{a} \hat{S}_{W}^{a} .
$$

Thus eigenfunctions of the boundary operator are classified according to the value of scalar product $\hat{L}_{W}^{a} \hat{S}_{W}^{a}$. The operator $\hat{S}_{W}^{a}$ looks like the nonrelativistic spin,

$$
\hat{S}_{W}^{a}=\frac{1}{2} \sigma^{a}
$$

and $\hat{\vec{L}}_{W}$ is the 3-dimensional angular momentum in spherical coordinates (in Cartesian frame it would be $\left.\hat{L}_{W}^{a}=\hat{L}^{a}=-i \epsilon^{a b c} x_{b} \partial_{c}\right)$.

Note, that despite the apparent resemblance $\hat{\vec{S}}_{W}$ is not the physical spin. Rotation (26) affects only the lower (right) components of 4-spinors and makes the actual spin operators for left and right fields look differently 1 .

However, one still may profit from the formal similarity of $S_{W}$ to spin. Let us introduce a fictitious operator of "total angular momentum" $\hat{J}_{W}=\hat{L}_{W}+\hat{S}_{W}$. Then the boundary operator in the work representation (29) may be written as

$$
\hat{B}_{W}=I+2 \hat{L}_{W}^{a} \hat{S}_{W}^{a}=I+\hat{J}_{W}^{2}-\hat{L}_{W}^{2}-\hat{S}_{W}^{2} .
$$

This beautiful formula is one of our main results and the key element of the further calculation. The reduction of spherical Dirac operator to momentum operators greatly simplifies all spectral expansions including that of the SBC-projector. Obviously the eigenfunctions of the operator (32) are conventional spherical spinors $\Omega_{j, l, m}$ and the corresponding eigenvalues of $\hat{B}_{W}$ are (see eqn. (15)),

$$
\lambda=j(j+1)-l(l+1)+\frac{1}{4}=\left\{\begin{array}{rll}
l+1, & \text { for } & j=l+\frac{1}{2} \\
-l, & \text { for } & j=l-\frac{1}{2} .
\end{array}\right.
$$

Explicit expressions for spherical spinors will be given in the next section.

\footnotetext{
${ }^{1}$ The true $4 d$ spin operator in Cartesian frame is $\frac{1}{2} \vec{\Sigma}$. After the rotation to work representation it becomes:

$$
\frac{1}{2} \vec{\Sigma}_{W}=\frac{1}{2} V_{W}^{\dagger} \vec{\Sigma} V_{W}=\frac{1}{2}\left(\begin{array}{rr}
\vec{\sigma} & 0 \\
0 & 2 \vec{n}(\vec{\sigma} \vec{n})-\vec{\sigma}
\end{array}\right)
$$




\subsection{Calculation of the projector}

Now we are going to calculate the 3 -dimensional projectors $\mathcal{P}_{3+1}^{ \pm}\left(q, q^{\prime}\right)$ defined by formulae (18). The eigenfunctions of the boundary operator $\hat{B}_{W}$ are the standard spherical spinors. In terms of the conventional spherical harmonics $Y_{l, m}$ they look as follows:

$$
e_{l+1}^{+}=\Omega_{l+\frac{1}{2}, l, k}=\left(\begin{array}{c}
\sqrt{\frac{j+k}{2 j}} Y_{l, k-\frac{1}{2}} \\
\sqrt{\frac{j-k}{2 j}} Y_{l, k+\frac{1}{2}}
\end{array}\right) \quad \text { and } \quad e_{l}^{-}=\Omega_{l-\frac{1}{2}, l, k}=\left(\begin{array}{c}
-\sqrt{\frac{j-k+1}{2 j+2}} Y_{l, k-\frac{1}{2}} \\
\sqrt{\frac{j+k+1}{2 j+2}} Y_{l, k+\frac{1}{2}}
\end{array}\right) .
$$

Substituting them into equations (18) we get for the projectors in the work representation (here $m=k-\frac{1}{2}$ and $\left.c_{l m}=\sqrt{(l-m)(l+m+1)}\right)$ :

$$
\begin{aligned}
\mathcal{P}_{W}^{+}(\hat{x}, \hat{y}) & =\sum_{l=0}^{\infty} \sum_{m=-l-1}^{l} \frac{1}{2 l+1} \times \\
& \left(\begin{array}{cc}
(l+m+1) Y_{l, m}(\hat{x}) Y_{l, m}^{*}(\hat{y}) & c_{l m} Y_{l, m}(\hat{x}) Y_{l, m+1}^{*}(\hat{y}) \\
c_{l m} Y_{l, m+1}(\hat{x}) Y_{l, m}^{*}(\hat{y}) & (l-m) Y_{l, m+1}(\hat{x}) Y_{l, m+1}^{*}(\hat{y})
\end{array}\right) ; \\
\mathcal{P}_{W}^{-}(\hat{x}, \hat{y}) & =\sum_{l=1}^{\infty} \sum_{m=-l}^{l-1} \frac{1}{2 l+1} \times \\
& \left(\begin{array}{ll}
(l-m) Y_{l, m}(\hat{x}) Y_{l, m}^{*}(\hat{y}) & -c_{l m} Y_{l, m}(\hat{x}) Y_{l, m+1}^{*}(\hat{y}) \\
-c_{l m} Y_{l, m+1}(\hat{x}) Y_{l, m}^{*}(\hat{y}) & (l+m+1) Y_{l, m+1}(\hat{x}) Y_{l, m+1}^{*}(\hat{y})
\end{array}\right) .
\end{aligned}
$$

The summation is a purely technical problem. At the end we get for the full 4-component projector (19):

$$
\left.\mathcal{P}_{W}(\hat{x}, \hat{y})\right|_{\hat{x}, \hat{y} \in S^{2}}=\frac{1}{2} \delta_{S^{2}}(\hat{x}-\hat{y})-i \gamma^{4} \mathcal{S}_{W}(\hat{x}, \hat{y})
$$

where $\mathcal{S}_{W}$ is the propagator of massless fermions in the work representation.

After rotating back to the Weyl representation (22) and substituting the fermion propagator $\mathcal{S}$ we get the explicit form of APS boundary condition on a sphere of radius $R$ :

$$
\begin{aligned}
&\left.\mathcal{P} \psi(\vec{x})\right|_{|x|=R}=\oint_{S^{2}}\left[\frac{1}{2} \delta_{S^{2}}(\vec{x}-\vec{y})+\frac{\not_{x}(\vec{x}-\vec{y})}{4 \pi|\vec{x}-\vec{y}|^{3}}\right] \psi(\vec{y}) d^{2} y= \\
& \oint_{S^{2}}\left[\frac{1}{2} \delta_{S^{2}}(\vec{x}-\vec{y})+\frac{\left(R^{2}-\vec{x} \vec{y}\right)}{4 \pi R|\vec{x}-\vec{y}|^{3}}\right] \psi(\vec{y}) d^{2} y=0
\end{aligned}
$$

This completes the calculation in 3-dimensional case. Now we are going to illustrate the mechanism that causes the appearance of the fermionic Green function by an example.

\section{The two-mode model}

Here we will demonstrate how the relation between the SBC-projector and fermion propagator arises at the level of a separated boundary harmonic. This may be done by studying the simple model with an almost trivial boundary operator.

Let us consider two-dimensional massless fermions living in half-plane $\xi<0$. Suppose that the boundary operator has a single eigenvalue $\lambda>0$ (actually this means that we

consider an individual mode of the boundary operator). Then the "Dirac operator" $\hat{\Lambda}$ is:

$$
\hat{\Lambda}=-i \gamma^{\xi} \partial_{\xi}+\hat{\lambda}=\left(\begin{array}{cc}
0 & \partial_{\xi} \\
-\partial_{\xi} & 0
\end{array}\right)+\left(\begin{array}{cc}
0 & \lambda \\
\lambda & 0
\end{array}\right)
$$


The Dirac equation $\hat{\Lambda} \psi=0$ has two solutions:

$$
\psi(\xi)=c^{-} \psi^{-}(\xi)+c^{+} \psi^{+}(\xi)=c^{-} \exp (-\lambda \xi)\left(\begin{array}{l}
0 \\
1
\end{array}\right)+c^{+} \exp (\lambda \xi)\left(\begin{array}{l}
1 \\
0
\end{array}\right) .
$$

The projectors onto positive and negative modes and unity operator are simple $2 \times 2$ matrices:

$$
P^{+}=\left(\begin{array}{cc}
1 & 0 \\
0 & 0
\end{array}\right) ; \quad P^{-}=\left(\begin{array}{cc}
0 & 0 \\
0 & 1
\end{array}\right) ; \quad I=P^{+}+P^{-}=\left(\begin{array}{cc}
1 & 0 \\
0 & 1
\end{array}\right) .
$$

The APS boundary condition in this case kills the positive mode that grows at $+\infty$ and has the form

$$
P \psi(\xi)=P^{+} \psi(\xi)=\left(\begin{array}{ll}
1 & 0 \\
0 & 0
\end{array}\right) \psi(\xi)=0
$$

Now let us turn to the Green function of operator (38). It is easy to check that

$$
\hat{\Lambda}^{-1}=S(\xi, \eta)=\left(\begin{array}{cc}
0 & \theta(\eta-\xi) e^{\lambda(\xi-\eta)} \\
\theta(\xi-\eta) e^{\lambda(\eta-\xi)} & 0
\end{array}\right) ; \quad \hat{\Lambda} S(\xi, \eta)=I \delta(\xi-\eta) .
$$

In order to establish a connection between the fermion propagator and SBC-projector we put both points $\xi, \eta$ onto the boundary, $\xi=\eta=0$, as it was done in (36), and multiply $S$ by $-i \gamma^{\xi}$ :

$$
-\left.i \gamma^{\xi} S(\xi, \eta)\right|_{\xi=\eta=0}=\left(\begin{array}{cc}
\theta(0) & 0 \\
0 & -\theta(0)
\end{array}\right)=\theta(0)\left(P^{+}-P^{-}\right)=\frac{P^{+}-P^{-}}{2} .
$$

Here we used the symmetric regularization and set $\theta(0)=\frac{1}{2}$. Comparison with (40) demonstrates that the projector onto the APS boundary conditions (41) may be expressed as

$$
P^{+}=\frac{1}{2} I-\left.i \gamma^{\xi} S(\xi, \eta)\right|_{\xi=\eta=0},
$$

that is exactly the one-dimensional version of Eq. (36).

We conclude that the result for the model is similar to that obtained by an exact calculation in the more realistic case. Actually the situation is quite general and the relation between the SBC-projector and fermionic Green function stays valid regardless of dimensionality of space, shape of the boundary and spinor representation. Indeed, the only simplification of our example is that the boundary operator had a single eigenvalue. Although the real spectra of boundary operators are much richer, our analysis remains true for every single isolated eigenfunction. Being valid for each of the spectral harmonics it must stay true for the functions as a whole.

\section{Conclusion}

Here is the brief summary of our results. We proposed the integral form of SBC for static even-dimensional bags and truncated SBC for odd-dimensional bags evolving in time. Then we performed the explicit calculation of SBC-projector for the 3-dimensional sphere. The projector has a simple and compact expression in terms of the massless fermion propagator. The analysis of a simple two-mode model of APS boundary conditions led to the same relation between the projector and propagator. 
The covariant form of our result may be obtained by transforming (36) to Weyl representation. Then the projector onto APS boundary conditions becomes:

$$
\mathcal{P}(\hat{x}, \hat{y})=V_{W} \mathcal{P}_{W}(\hat{x}, \hat{y}) V_{W}^{\dagger}=\frac{1}{2} \delta_{\partial B}(\hat{x}-\hat{y})-i\left(\hat{\gamma} \hat{n}_{x}\right) \mathcal{S}(\hat{x}, \hat{y}),
$$

where vectors $\hat{x}, \hat{y} \in \partial B$ and $\hat{n}_{x}$ is the outward normal to the boundary at point $\hat{x}$. Here $\mathcal{S}$ is the conventional propagator of massless fermions. This expression does not depend on parametrization of $\gamma$-matrices and choice of coordinates.

The relation between the SBC-projector and massless fermion propagator (45) is covariant and does not depend on representation of spinors. Therefore the integral formulation of spectral boundary conditions looks more practical than the original "constructive" definition that required the special choice of coordinates $\gamma$-matrices etc. Moreover, the result (45) appears to be universal and suitable for boundaries of arbitrary shape in any dimension. It is not difficult to prove this in general starting from the two-mode example of Section 3 .

\section{Acknowledgements}

In conclusion we are glad to thank D. Laenge, A. Kirchberg, K. Kirsten and M. Santangelo for discussions on various aspects of APS boundary conditions. A. Abrikosov expresses his gratitude for the DAAD sponsorship that made possible the present collaboration. His work was also partially supported by RFBR grants 05-02-17464 and 06-02-16905.

\section{References}

[1] A. Chodos, R. L. Jaffe, C. B. Thorn, and V. F. Weisskopf, Phys. Rev. D9 (1974) 3471.

[2] S. Duerr, A. Wipf, Nucl. Phys. B443 (1995) 201.

[3] G. Esposito, K. Kirsten, Phys. Rev. D66 (2002) 085014.

[4] S. Théberge, A. W. Thomas, G. A. Miller, Phys. Rev. D22 (1980) 2838; Phys. Rev. D23 (1981) 2106(E).

[5] M. F. Atiah, V. K. Patodi, I. M. Singer, Math. Proc. Camb. Phil. Soc. 77 (1975) 43.

[6] T. Eguchi, P. B. Gilkey, A. J. Hanson, Phys. Rep. C 66 (1980) 213; H. Roemer, P.B. Schroer, Phys. Lett. B21 (1977) 182; A. J. Niemi, G.W. Semenoff, Phys. Rep. 135 (1986) 99; Nucl. Phys. B293 (1987) 559.

[7] A. J. Niemi, G. W. Semenoff, Phys. Rev. D32 (1985) 471; R. Musto, L. O’Raifeartaigh, A. Wipf, Phys. Lett. B175 (1986) 433.

[8] A. A. Abrikosov jr., Journ. of Phys. A39 (2006) 6109, hep-th/0512311.

[9] M. Ninomiya, C. I. Tan, Nucl. Phys. B245 (1985) 199; Z. Q. Ma, Journ. of Phys. A19 (1986) L317; P. Forgacs, L. O’Raifeartaigh, A. Wipf, Nucl. Phys. B293 (1987) 559 . 
[10] C. Jayewardena, Helv. Phys. Acta 61 (1988) 636.

[11] A. A. Abrikosov jr., Int. Journ. of Mod. Phys. A17 (2002) 885; hep-th/0212134. 